\title{
College Students' Quality Development to Create and Manage Files
}

\author{
Wang Juncheng \\ Energy and Power Engineering, Northeast Electric Power University, Jilin, Jilin, China
}

\begin{abstract}
Students Quality Development CYL Central Committee, the Ministry of Education, the National Federation of the joint implementation of the program has been carried out for many years, most colleges and universities in the plan also gives enough attention and increases the overall quality of students records on file at the college, but most colleges and universities for the current file on the overall quality of college students a records management perspective, there are still many imperfections, specially built for this it is necessary to expand the quality of a set of files on college students, to stimulate students' self enthusiasm for learning, improve their overall quality, which can achieve the implementation of the Quality Development plan objective.
\end{abstract}

Keywords - Students' Quality Development Plan, records management, comprehensive quality

\section{高校大学生素质拓展档案的建立与管理}

\author{
王俊成 \\ 东北电力大学能源与动力工程学院, 吉林, 吉林, 中国
}

\begin{abstract}
摘 要 共青团中央、教育部、全国学联所联合实施的大学生素质拓展计划已经开展多年, 大部分高校对该计划也给于了足够的 重视, 并在大学生档案中增加了关于大学生综合素质的记录, 但就目前多数高校对于大学生档案中关于综合素质一项记录管理来看, 还存在着很多不完善之处, 对于此有必要专门建立出一套关于大学生素质拓展的档案, 以激发大学生自我学习热情,提高其综合素质, 从而能够达到大学生素质拓展计划实施的目的。
\end{abstract}

关键词 大学生素质拓展计划, 档案管理, 综合素质

1. 引言

大学生素质拓展计划能够给大学生带来实践活动综合 能力的提高, 是以社会实际需求为导向的一种培养策略。 因此，对于大学生素质拓展计划所实施培训内容及结果有 必要记录于档案之中，让大学生能够充分重视这方面能力 提高的同时, 也能够给于大学生综合素质能力一定的凭证, 让走向社会之后的大学生能够被用人了解到更多, 在他们 之间起到一种桥梁性的作用。

\section{2. 大学生素质拓展计划解构}

\section{1 职业设计环节}

职业设计环节是大学生拓展计划当中基础性环节, 它 的重要性不言而喻, 他主要包含了大学生价值取向、职业 规划、心里素质、品质意志、责任意识、社交技能等方面
的培养。具体而言, 在职业设计环节当中, 大学身高素质 拓展计划主要强调从五个层次对大学生素质进行拓展: 职 业理想及职业人生规划能力; 成长发展经历认知程度; 品 德修养、心理素质以及意志力水平; 社会责任意识、价值 取向的积极度; 实践能力、创新意识及创业精神。

\section{2 素质训练环节}

素质训练环节, 是开发大学生素质的基本原动力。它 的训练是以人力资源思维为主导, 将素质训练项目模块化, 进行具体有针对性目标的训练。具体而言, 这些素质训练 模块对应的培养目标如下：思想政治道德修养模块 $\rightarrow$ 思想 道德素质目标; 社会实践与志愿服务模块 $\rightarrow$ 实践能力、适 应能力、道德修养; 学术科技与创新创业模块 $\rightarrow$ 创新能力 
和科学精神; 文化艺术与身心发展模块 $\rightarrow$ 人文精神和人格 培养; 社团活动与社会工作模块 $\rightarrow$ 适应能力、管理能力、 素质修养; 技能培训及其他活动模块 $\rightarrow$ 各种技能和特长。

\section{3 评价体系环节}

对于大学生素质拓展技术这个评价体系环节, 指的是 对于大学生实施素质拓展培训之后所必须设置一些评价性 机构、评价性内容以及流程和评价证书等。对于这些而言, 相对较为的繁琐, 但粗略的看, 可将这些最终归为具有合 理评价机构对参与素质拓展学生进行合理而规范的素质评 价。

从大学生素质拓计划的基本构成, 也可以看出大学生 所需拓展素质的基本层面, 而在建立档案的时候就必须按 照这些方面内容和原则展开, 并进行相应的管理, 以此方 能够使得建立的档案与素质拓展计划相互呼应, 起到应有 的作用。

\section{3. 大学生素质拓展档案主要内容}

在高校建立大学生素质拓展档案具有的现实意义不言 而喻, 而从当今高校对于大学生综合素质记录管理情况上 看, 建立这方面的档案需求便体现的更加的迫切。基于大 学生素质拓展计划的内容结构要求, 对于大学生素质档案 内容上提出以下几方面建议:

\section{1 建立政治学习记录}

政治学习是十分必要的, 因此, 在在大学生素质拓展 档案当中, 此类记录应列为第一项重要记录。该记录应记 载大学生在学生干部培训班、团校、党校等思想阵地的学 习情况。以能够充分反映出大学生政治学习水平。

\section{2 建立社会实践活动记录}

社会实践能力是大学生素质拓展计划培养的主要目标 之一。因此该记录应该详实、清哳地记录起来。应包括能 够真实反映大学生参加的社会实践活动及取得的成绩。包 括如支教、支边或是各类周末实践活动及志愿者活动。

\section{3 建立学术科技记录}

这部分应当记载大学生在大学期间在科研方面所取得 的成绩, 包括取得的发明创造、发表的科技学术论文及在 科技竞赛中获取的成绩等等。其能够反映出大学生在素质 拓展中的创新能力水平。

\section{4 建立社团工作记录}

社团工作记录能够反映出大学生社会交往能力, 是高 校大学生素质拓展计划当中重要 的一环。档案中这部分记 录应主要记载大学生在高校期间, 主要参加的社团及担任 学生组织的相应职务。

\section{5 建立实习成绩记录}

这部分实习成绩记录不仅能够反映出大学生对大学期 间知识的把握程度, 更加能够透视出大学生的即时应变能 力。这部分应主要记载大学生在大学期间从从事过的实习、 实训等展开情况及所获取的评价、成绩。

3.6 建立资格证书记录

该部分记录主要记载大学生在学期间获得的各类等级 证书及资格资质证书等。大学生素质拓展档案将这些证书 归类于档案之中, 有利于技能水平的真实性, 防止造假证 书出现。

\section{7 建立身心素质记录}

这部分记录包含身体素质及心理素质。它是素质拓展 计划落实于大学生个体一个最为直观的展现, 通过素质拓 展后记录, 开拓了大学生毕业之后所在用人单位人对于其 认识程度。该记录, 应详实的记载在素质拓展中大学生这 两方面素质变化的真实情况。

\section{4. 高校建立大学生素质拓展档案的意义}

大学生素质拓展计划的目的是为了拓展大学生综合素 质水平, 这也是该计划最为重要意义所在之处。而如何能 够将这种拓展成果转化为激发大学生自我学习、努力完善 提高自身素质修养的动力, 就变得及其重要。而通过对于 大学身素质拓展档案的建立, 合理的管理就是巩固这种成 果极为有效的手段, 因此在高校建立大学生素质拓展档案 便具有了多方面的重要价值。

\section{1 大学生素质拓展档案的建立有利于用人单位对大学生 的选择}

从传统的视角看, 通常透视出一个大学生能力水平的, 往往是一纸成绩单, 或某方面证书。这些方面的成果展示, 当然无可厚非, 它可以从某一个方面展示出大学生的能力 水平。但是这些过于狭险化的内容, 难以全面的展示出大 学生的综合水平, 或者可以说是发展潜力。这些情况, 在 当今大学生就业当中不仅给用人单位在录用人才时候造成 困难, 同时对于大学生自身找准自身的位置, 去应聘合理 
适合自身的岗位也是相当不利的。而通过大学生素质拓展 档案的建立, 从职业人生计划认知度到具体的实践活动的 表现, 再到高校相关机构的评估, 能够全然的将这些抽象 化的素质表现跃然于纸上。这些方面的综合表现, 不再是 以个人主观喜好或目的为准绳, 而是以一个客观公正的角 度将大学生动手能力、交往能力、创新能力等等都汇集于 档案之中, 最终让社会能够对于不同类型的大学生作出合 理的取舍, 而对于大学生而言适合于自身岗位选择了自己, 对自身的发展也是十分有利的。

\section{2 大学生素质拓展档案的建立有利于激发大学生自我提 高的热情}

在当今的高校提倡的思想及素质教育当中, 多数采用 的方式依然是以课程学分制来要求大学生参与其中, 而这 种较为传统的授课方式一方面大学生的参与度不高, 更为 重要的是经过课程教育之后, 能够对大学生起到的作用也 难以预见。而通过建立大学生素质拓展档案的方式, 通过 记录大学生在参与到素质拓展实践中的表现, 最后将这些 都归于档案当中, 能够让大学生在认识到其重要性的同时 更加能够激发他们自我学习、提高的热情。通过这样的方 式, 最后从提高学生自身积极性角度, 能够全面提高他们 的各方面学习成果, 最后能够对于其自身综合素质的提高 产生积极的影响。

\section{5. 建立大学生素质拓展档案应注意的问题}

大学生素质拓展档案的建立首先应以大学生在素质拓 展活动中真实表现为基础原则, 档案的真实性与否事关学 生的声誉以及后续单位用人时的选拔。高校必须以真实性 原则进行有力的把握, 方能够让大学生素质档案越来越具 有权威性。而在真实性原则基础之上, 大学生素质拓展档 案的建立, 还必须应该具有科学性。因为大学生素质评价 工作, 并不是一项简单的工作, 不能以考试成绩来衡量, 它是一项系统性的项目, 高校在建立档案之初就应在以真 实为基础, 进行方方面面的考量。除此之外, 公正性原则、 实用性原则是大学生素质档案的存在和得以发展的基础, 没有了公正性原则这一切都将毫无存在价值。而实用性原 则同样如此, 没有了能够可与实际管理所相契合的性质同 样存在意义不大。

在对大学生素质拓展档案上述这些基本原则进行把握 之下, 还有一些管理上的制度问题需要加以注意:
第一, 归档制度需规范。大学生素质拓展档案的管理 部门应于高校各个素质拓展相关人员紧密联系, 保证各个 环节之间能够流畅运行保证能够定时、定向、定内容的做 好收集归档工作。这些素质拓展相关人员包括了院系的素 质拓展负责人、辅导员、勤工俭学负责人等等, 这些都是 参与到大学生素质拓展计划当中所必须经由关键性人员, 对于与这些人员的紧密联系能够使归档工作能够呈辐射状 态, 让档案中所需要的记录内容得到及时的收集与反馈。

第二, 建立档案收集标准体系。目前来说, 高校在学 生档案管理方面, 除了学生成绩档案收集及管理工作实施 较为严格的管理制度之外, 对于这之外信息收集管理则处 在松散状态, 没有相应的管理制度体系可供参考, 因此也 就处在管理较为随意的状态中。而这些情况也是导致当今 档案中关于素质拓展的内容各个高校之间、或是高校之内 都没有可比性, 实施上也就失去了存在价值。因此, 需要 对于大学生素质拓展档案建立起一套对应的标准体系, 能 够使得各个高校之间都能够产生对比差异, 让素质拓展档 案起到应有的作用。

第三, 更新管理理念。目前高校学生档案的管理人员 主要都是受到院系学生管理部门指定而兼职的, 因为兼职 人员在高校还有其它主要工作要完成, 这就导致了档案管 理工作往往是在学生毕业之后应急处理。而对于大学生素 质拓展档案来说, 这种滞后性的管理方式, 明显不适用, 不能够及时归档一些学生信息, 让大学生素质拓展档案失 去应有的效应。对于此, 高校应该更新管理理念, 做到科 学管理, 及时管理, 从而使大学生素质拓展档案得到高效 应用。

\section{参考文献(References)}

[1] Wang Yupeng, Feng Yan Lee, from college students to see college students employment file problems and countermeasures. Lantai World, 2009 (20)

[2] Liu Yanhui role of archival information resources to expand the students' quality. Yunmeng learn Journal, 2009, (4).

[3] Li Shuyan strengthen college students' employment student records management services. Yunnan file, 2009, (9).

[4] Qiurui Ling, Liu Kerong. University archives Undergraduate Research Competitiveness and Employment. Lantai World, 2012 (20). 